\title{
Activated carbon from coal tar pitch and furfural for the removal of $\mathbf{p}$ - nitrophenol and m-aminophenol
}

Bilyana Petrova ${ }^{\mathrm{a}}$, Boyko Tsyntsarski ${ }^{\mathrm{a}}$, Temenuzhka Budinova ${ }^{\mathrm{a}}$, Nartzislav Petrov ${ }^{\mathrm{a}}$, Leticia F. Velasco $^{\mathrm{b}}$, Conchi O. Ania ${ }^{\mathrm{b}}$

${ }^{a}$ Institute of Organic Chemistry, Bulgarian Academy of Sciences, Sofia 1113, Acad.G.

Bonchev str., BL. 9, fax (359-2) 7002 25, e-mail: goriva@orgchm.bas.bg, Bulgaria

${ }^{\mathrm{b}}$ Instituto Nacional del Carbon, CSIC, Apartado 73, 33080, Oviedo, Spain

\begin{abstract}
Adsorption of m-aminophenol and p-nitrophenol from aqueous solution on activated carbon synthesized on the base of industrial by product (coal tar pitch) and furfural was investigated. The adsorption capacity of the activated carbon and its oxidized modification is related to the surface area and composition of the prepared materials, as well as to the nature of the adsorbents. Despite the moderate porosity, the prepared materials display adequate adsorption capacity towards the investigated aromatic compounds. Both adsorbates show higher affinity towards the hydrophobic carbon, confirming that the retention mechanism occurs via nonspecific interactions between the electronic density of the adsorbent and the aromatic pollutants. Electrostatic interactions may also appear depending on the solution $\mathrm{pH}$ and the charge distribution of the carbons; we have observed that this parameter has a strong influence on the final performance of the oxidized carbon, where repulsive interactions that reduced the uptake of the carbon were found to appear within a larger $\mathrm{pH}$ range.
\end{abstract}

Keywords: coal tar pitch; furfural; activated carbon; adsorption; p-nitrophenol; maminophenol

Corresponding author. Present address: Institute of Organic Chemistry, Acad.G.Bonchev str., bl.9, 1113 Sofia, Bulgaria. Tel.: +359 2 9606145; fax: +3598700225

E-mail address: goriva@orgchm.bas.bg (T. Budinova) 


\section{Introduction}

As a result of the extensive development of industrial activities in the last 100 years, large amounts and rich variety of chemical wastes and pollutant are being thrown into water resources. Chemical pollution of surface waters presents a threat to the aquatic environment with negative effects on ecosystems (acute and chronic toxicity to aquatic organisms, accumulation in the ecosystems and loses of habitats and biodiversity, due to low biodegradability) as well as on human health (carcinogenic and mutagenic effects). The occurrence of large concentrations of toxic pollutants in wastewater has become an important environmental issue, and very often small amounts of a large number of synthetic organic compounds (including phenol and its derivatives, pesticides, aliphatic and aromatic hydrocarbons, dyes, surfactants) are detected in water streams.

Phenolic compounds are prevalent in many industrial wastewaters, including those from the manufacture of insecticides, herbicides, dyes, pulp and paper, and other synthetic chemicals. Along with phenol itself, nitro- and aminophenols are most abundant phenolic compounds in industrial wastewater. They are widely used in the manufacture of explosives, pharmaceuticals, pesticides, pigments, and dye and rubber chemicals [1-3]. In recognition of its toxicity, high mobility in the environment and low biodegradability, the US and European Environmental Protection Agencies (EPA and EEA) have included these compounds in their lists of priority pollutants to be monitored in industrial effluents [4-6]. In this regard, adsorption technology has become a well established and powerful technique for water treatment, being activated carbons the most effectively used adsorbents due to their adequate porous and chemical features [7].

Our earlier investigations focused on the synthesis of porous carbons based on polyolefine wax, furfural and mixtures of tar from carbonization of agricultural by-products and furfural [8-10]. We have observed that the chemical composition of the precursor strongly influences the structure and properties of the obtained adsorbent. For instance, the carbonizate - before activation - obtained from pyrolysis of a specific raw material containing dominantly aromatic compounds (such as biomass-derived tar), is characterized by a dense structure and weak reactivity towards steam activation. On the contrary, when furfural is used as precursor, the final carbon material is less dense and more reactive, due to the insertion of oxygen in the carbon skeleton that favors the formation of pores during the activation process. In contrast, when mixtures of coal tar pitch and furfural in different proportions are used as precursors, 
the synthesized carbons displayed physicochemical and nanoporous features that can be tailored by an adequate composition of the precursor mixture [11].

The aim of our research was to investigate the adsorptive performance of synthetic carbons obtained from polymerization of a mixture of coal tar pitch and furfural, for removal of pnitrophenol and m-aminophenol from aqueous solution. The m-aminophenol (MAP) and pnitrophenol (PNP) were chosen as representative phenols due to electron-donating and -withdrawing groups, respectively. For this purpose, a nanoporous carbon obtained from a precursor mixture with ratio coal tar pitch to furfural 55:45 was selected as adsorbent. This material, initially characterized by a large amount of oxygen-containing groups of basic nature- was oxidized to incorporate further oxygen functionalities of acidic nature. The adsorption capacity of both nanoporous carbons for the removal of two phenolic pollutants from diluted aqueous solutions was evaluated and discussed in terms of the porous structure and chemical composition of the adsorbents.

\section{Materials and methods}

\subsection{Materials}

A mixture of coal tar pitch and furfural (55:45 wt.\%) was treated with concentrated $\mathrm{H}_{2} \mathrm{SO}_{4}$ (drops of $\mathrm{H}_{2} \mathrm{SO}_{4}$ were added under continuous stirring) at $120{ }^{\circ} \mathrm{C}$ until solidification. The obtained solid product was heated at $600{ }^{\circ} \mathrm{C}$ in a covered silica crucible with a heating rate of $10{ }^{\circ} \mathrm{C} \mathrm{min}{ }^{-1}$ under nitrogen atmosphere. The carbonized solid was further submitted to steam activation at $800^{\circ} \mathrm{C}$ for $1 \mathrm{~h}$ (carbon $\left.\mathrm{A}\right)$.

The sample was submitted to a wet oxidation treatment in nitric acid, for incorporating oxygen-based functionalities on the carbon matrix. The oxidation was performed according to the following procedure: about $10 \mathrm{~g}$ of the sample was treated with $100 \mathrm{ml} 10 \% \mathrm{HNO}_{3}$ and boiled for $1 \mathrm{~h}$. Afterwards, the sample was thoroughly washed in distilled water, dried at $105^{\circ} \mathrm{C}$ and kept in dessicator until used. The oxidized carbon was labeled as carbon B. The particle sizes were between $0.2-0.3 \mathrm{~mm}$.

\subsection{Methods}

\subsubsection{Physico-chemical characterization: XRD and SEM}

Powder X-ray diffraction spectra were collected within the $2 \theta$ range from 5 to $80^{\circ}$, with a constant step $0.02^{\circ}$, on Bruker D8 Advance diffractometer with $\mathrm{Cu} \mathrm{K} \alpha$ radiation and 
LynxEye detector. The morphology of the activated carbon was examined by means of a scanning electron microscope (JEOL-Superprobe 733).

\subsubsection{Pore structure analysis}

Nanotexture of the synthesized carbon materials was characterized by $\mathrm{N}_{2}$ adsorption at $196^{\circ} \mathrm{C}$, carried out in an automatic volumetric apparatus (ASAP 2020 from Micromeritics). Before the experiments, the samples were outgassed under vacuum at $120^{\circ} \mathrm{C}$ for overnight. The isotherms were used to calculate specific surface area $S_{B E T}$, total pore volume $V_{T}$, micropore volume $\mathrm{W}_{\mathrm{o}}$ (using the DR formulism) [12].

\subsubsection{Oxygen-containing functional groups.}

The amount of oxygen-containing functional groups with increasing acidity was determined by Boehm's method of titration with basic solutions of different base strengths $\left(\mathrm{NaHCO}_{3}\right.$, $\mathrm{Na}_{2} \mathrm{CO}_{3}, \mathrm{NaOH}, \mathrm{C}_{2} \mathrm{H}_{5} \mathrm{ONa}$ ) [13]. For this purpose the samples were agitated for at least $16 \mathrm{~h}$ with $0.05 \mathrm{~N}$ solutions of four bases. The amount of $\mathrm{Na}+$ ions remaining in the solution is determined by adding an excess of standard $\mathrm{HCl}$ and back-titration. The basic groups contents of the oxidized samples are determined with $0.05 \mathrm{~N} \mathrm{HCl} \mathrm{[14].}$

\subsubsection{Determination of $\mathrm{pH}$}

The procedure is as follows: $4.0 \mathrm{~g}$ of carbon (ground, not dried) is weighted into a 250-ml beaker and $100 \mathrm{ml}$ of water is added. The beaker is covered with a watch glass and heated to boiling temperature for $5 \mathrm{~min}$. The mixture is set aside and the supernatant liquid is poured off as hot as possible but not below $60^{\circ} \mathrm{C}$. The decanted portion is cooled to room temperature and is measured to the nearest $0.1 \mathrm{pH}$.

\subsection{Adsorption measurements}

Phenolic substances (m-aminophenol and p-nitrophenol) were obtained from Merck (99\% purity). The phenolic solutions were prepared in non-buffered distilled water in the concentration range of 100-250 $\mathrm{mg} \mathrm{l}^{-1}$. About $100 \mathrm{mg}$ of activated carbons were mixed with $50 \mathrm{ml}$ of phenolic solution of the desired concentration, and the suspensions were shaken for 2 hours. The samples were filtered and the equilibrium concentrations of the phenolic compounds remaining in solution were determined spectrophotometrically (286 nm for maminophenol, and $316 \mathrm{~nm}$ for m-nitrophenol) in a Pfaro $300 \mathrm{UV}$ spectrometer. Each determination is performed in triplicate. The amount adsorbed $\left(\mathrm{q}_{\mathrm{e}}\right)$ per unit mass of adsorbent was evaluated from the equation: $\mathrm{q}_{\mathrm{e}}=\mathrm{V}\left(\mathrm{C}_{\mathrm{o}}-\mathrm{C}_{\mathrm{e}}\right) / \mathrm{m}$, where $\mathrm{V}(\mathrm{ml})$ is the volume of the 
solution, $\mathrm{m}(\mathrm{g})$ the mass of the adsorbent, and $\mathrm{C}_{\mathrm{o}}$ and $\mathrm{C}_{\mathrm{e}}$ are the initial and equilibrium concentrations of the aromatic compound in the liquid phase $\left(\mathrm{mg} \mathrm{l}^{-1}\right)$, respectively. The free $\mathrm{pH}$ of the solutions during adsorption was in the range 5-6.

To investigate the effect of solution $\mathrm{pH}$ on the adsorption capacity of the studied carbons, different solution $\mathrm{pH}$ were obtained by adding $\mathrm{NaOH}$ or $\mathrm{HCl}$ to the stock solution containing the aromatic compound.

\section{Results and discussion}

\subsection{Materials synthesis and characterization}

Earlier studies carried out in our research group have shown that furfural resin is a suitable oxygen-containing raw material for the production of carbon adsorbents with a large number of oxygen-containing groups on the surface [9]. Furfural is an accessible and cheap material that accelerates the polymerization processes in the mixtures. Indeed, carbon adsorbents with different structure and properties have been prepared from mixtures of furfural and biomassderived tars, while optimizing the operating conditions (including the composition of the mixtures, activation reagents, pre-treatment of the precursors, etc.) [10, 11].

In this work, coal tar pitch was selected as the carbon precursor. The elemental analysis of the coal tar pitch (Table 1) confirms that the amount of oxygen containing structures is not high, whereas the high $\mathrm{C} / \mathrm{H}$ ratio indicates the presence of considerable amount of aromatic species in the pitch. The addition of furfural to the coal tar pitch, used in the precursor mixture, aims to incorporate a reactive structure in the carbon matrix, capable of inducing polymerization and polycondensation reactions, that will facilitate the solidification of the mixture. The resulting solid product was carbonized and submitted to steam activation to produce a suitable porous adsorbent.

Table 1. Chemical composition and $\mathrm{pH}$ value of the coal tar pitch and the synthesized activated carbons (wt.\%)

\begin{tabular}{llllllll}
\hline Sample & Ash & Volatiles (daf) & C & H & N & S & O \\
\hline Coal tar pitch & - & - & 90.90 & 4.95 & 0.90 & 0.50 & 2.75 \\
\hline Carbon A & 0.8 & 1.9 & 90.81 & 0.82 & 0.75 & 0.51 & 7.11 \\
\hline Carbon B & 1.5 & 7.7 & 86.77 & 1.02 & 1.30 & 0.56 & 10.35 \\
\hline
\end{tabular}


After carbonization and activation of the mixture, a carbonaceous solid is obtained with a final yield of $58 \mathrm{wt} \%$. Analysis of the chemical composition of carbon A shows that, along with the prevailing content of aromatic structures in the pitch, the resulting material has relatively large oxygen content (Table 1). This corroborates that inserting oxygen in the carbon precursor (i.e. furfural) leads to the formation of oxygen containing structures on the surface of the final product.

The X-ray diffraction pattern of the synthesized nanoporous carbon (sample A) is shown in Figure 1 as an example. The pattern consists of two intense and considerably narrow peaks located at $24.5^{\circ}$ and $43.5^{\circ}$, corresponding to the (002) and (100) crystalline graphite. These peaks corroborate the presence of a porous network, associated to the typical turbostratic structure of carbon materials. The sharp and intense peaks suggest that the carbon has a considerably ordered structure and a non negligible degree of graphitization, despite the interlayer distance of graphitic sheets was higher than those in graphite.

The typical morphology of the synthetic nanoporous carbon can be seen from the SEM micrograph presented on Figure 2. The material consists of small particles which form aggregates with similar dimensions which shows a good homogenization.

The textural properties of the selected nanoporous carbon are shown in Table 3. In a previous work about the effect of the ratio furfural:coal-tar-pitch in the carbon precursor, we have observed that the furfural content has a strong effect on the porosity of the resulting carbon [11]. As a general rule, low proportions of furfural give rise to activated carbons with a narrow microporosity, which is gradually opened in favor of large micropores and mesopores as the furfural content rises. Notwithstanding, the carbon yield also decreases sharply with rising the furfural content, due to the reactivity of precursor mixture. As above-mentioned, in this study we have selected a furfural content of ca. $45 \mathrm{wt} . \%$ in the precursor mixture. The resulting carbon still shows quite developed porous features, while keeping a large carbon yield (ca. 53\%) in the synthesis, which allows to synthesize large amounts of carbon per batch.

The synthesized carbon also displays strong alkaline character, as inferred from the $\mathrm{pH}$ value (Table 2). After oxidation in nitric acid, as expected, there is an enhancement in the overall oxygen content, accompanied by a fall of the $\mathrm{pH}$ value, confirming the different nature of the surface functionalities, created upon oxidation. The surface functionalities were further characterized by the Boehm titration (Table 2). Data shows that the surface chemistry of carbon $\mathrm{A}$ is comprised of carbonyl and phenolic functionalities which render a basic character 
to the adsorbent. In contrast, even if the groups of the parent carbon are well preserved, oxidation with nitric acid caused the formation of acidic groups (carboxyl and lactonic structures), which are responsible for the decrease in the carbon $\mathrm{pH}$ (ca. $4 \mathrm{pH}$ units fall). Along with the incorporation of acidic surface groups, a twofold decrease in the amount of basic groups was also observed after oxidation of carbon A. This is attributed to the incorporating of the created groups at the edges of the basal planes in the graphene layers, thereby reducing the capacity of these sites to accept $\mathrm{H}^{+}$ions (acting as Lewis base).

Table 2. Data corresponding to Boehm's titration for the quantification and identification of the oxygen surface groups on the activated carbons [meq $\left.\mathrm{g}^{-1}\right]$.

\begin{tabular}{lllllll}
\cline { 2 - 6 } Sample & Carboxylic & Lactonic & Phenolic & Carbonyl & Basic groups & $\mathbf{p H}$ \\
\hline Carbon A & BDL* & BDL & 0.210 & 1.356 & 0.778 & 8.1 \\
\hline Carbon B & 0.109 & 0.239 & 0.456 & 1.767 & 0.330 & 3.7 \\
\hline
\end{tabular}

*BDL - below detection limit

Table 3. Results obtained from Langmuir equation applied to the adsorption isotherms of phenolic compounds on the studied activated carbons.

\begin{tabular}{lccccc}
\hline Sample & & $\begin{array}{c}\mathbf{Q}_{\mathbf{0}} \\
{\left[\mathbf{m g ~ g}^{-\mathbf{1}}\right]}\end{array}$ & $\begin{array}{c}\mathbf{b} \\
{\left[\mathbf{l ~ m g}^{-\mathbf{1}}\right]}\end{array}$ & $\begin{array}{c}\mathbf{b Q}_{\mathbf{0}} \\
{\left[\mathbf{l} \mathbf{g}^{-\mathbf{1}}\right]}\end{array}$ & $\mathbf{R}^{\mathbf{2}}$ \\
\hline Carbon A & MAP & 110 & 0.53 & 58.7 & 0.994 \\
\hline Carbon A & PNP & 132 & 0.22 & 29.0 & 0.999 \\
\hline Carbon B & MAP & 85 & 0.48 & 42.7 & 0.969 \\
\hline Carbon B & PNP & 97 & 0.11 & 10.5 & 0.985 \\
\hline
\end{tabular}

In this regard, carbon A was prepared with a moderate proportion of furfural in the precursors' mixture (45 wt.\%), being characterized by a moderate BET surface area and an well developed microporosity (Figure 3).

These porous features should be ideally adapted for the removal of aromatics from aqueous phase. Oxidation brought about a decrease in the porous features of the carbon (likely due to the boiling step during oxidation), and the fall in the micropore volume accounts for only $16 \%$. Summarizing, we have prepared two carbon materials with varied surface chemistry 
(oxygen-enriched materials) but different basicity. It is interesting to point out that such combination of chemical features is rather unusual in porous carbons, only been described in the literature for carbons prepared from biomass precursors [15-17]. These activated carbons were further used to investigate the removal of organic pollutants ( $\mathrm{m}$-aminophenol and $\mathrm{p}$ nitrophenol) from aqueous phase.

\subsection{Adsorption from solution}

Initially, the adsorption kinetics of both aromatics on the studied carbons were evaluated, in order to assess the time, needed for equilibrium before determining the maximum adsorption capacity of each adsorbent. The kinetics of adsorption of MAP and PNP from aqueous solution were studied for various concentrations ranging from 100 to $250 \mathrm{mg} / \mathrm{L}$ for both carbons; the results obtained for $200 \mathrm{mg} / \mathrm{L}$ are shown in Figure 4 as an example. The obtained kinetics of adsorption shows the well-know pattern of aromatic compounds described in the literature, with a fast uptake of both compounds at the initial stage of the adsorption process (short times), equilibrium being reached within $40 \mathrm{~min}$ in all cases [18-20]. Moreover, the amounts adsorbed show smooth continuous curves in all the cases, with a well defined saturation plateau. This behavior is attributed to the decrease in the number of available adsorption sites on the carbon surface as the adsorption proceeds and the first molecules of adsorbate are retained.

Comparatively, the uptake is slightly faster in the most hydrophobic sample (carbon A) for both pollutants, while for a specific carbon there does not seem to be a kinetically favored retention of MAP or PNP. Since both compounds show rather close solubility in water (i.e., 2.6 and $1.7 \mathrm{~g} / 100 \mathrm{~g} \mathrm{H}_{2} \mathrm{O}$ for MAP and PNP, respectively), the similarities in the rate of adsorption on a specific carbon are somewhat expected. On the other hand, the faster uptake on the hydrophobic carbon -compared to carbon B- is rather surprising; it suggests that besides the enhanced wet ability of the carbon after oxidation, kinetic restrictions for the accessibility of the aromatic compound to the adsorption sites arise, probably due to water also competing for these sites.

The adsorption isotherms of MAP and PNP on both activated carbons are depicted in Figure 5. All of them belong to type $\mathrm{L}$ of Giles classification, indicating that adsorption proceeds by the formation of a monolayer in the range of concentrations used [21]. The retention of both PNP and MAP is very similar for a specific carbon, although the uptake is favored in hydrophobic carbon A (slope of the isotherm at low concentration is steeper). The 
higher uptake of PNP, compared to MPA, is in good agreement with previous works reported in the literature $[22,23]$. The experimental data was fitted to the Langmuir equation [24]:

$\mathrm{Q}_{\mathrm{eq}}=\mathrm{Q}_{\mathrm{o}} \mathrm{b} \mathrm{C}_{\mathrm{eq}} /\left(1+\mathrm{b} \mathrm{C}_{\mathrm{eq}}\right)$

where $C_{e q}$ is the equilibrium pollutant concentration remaining in solution after adsorption (mg/l), $Q_{e q}$ is the amount of pollutant bound to the adsorbent $(\mathrm{mg} / \mathrm{g}), Q_{o}$ is the maximum amount of the pollutant per unit weight of adsorbent $(\mathrm{mg} / \mathrm{g})$, and $b$ is a constant related to the affinity of binding sites $(1 / \mathrm{mg})$.

On the other hand, it is interesting to remark that despite their moderate porosity development, activated carbons prepared from mixtures of furfural and coal tar pitch, present adequate adsorption capacities towards MAP and PNP, comparable to those reported in the literature for activated carbons with higher surface areas [25-27].

These parameters, together with the relative affinity of the adsorbates towards the surface of the adsorbents - parameter $b \mathrm{Q}_{0}$, and the correlation coefficients, are complied in Table 3 . The very good fittings, obtained for all the samples, indicate that Langmuir model is capable of predicting the adsorptive behaviour of PNP and MAP in these carbons. The adsorption capacities of the both pollutants in the studied carbons confirm the observed trend with the hydrophobic character of the carbon. It is also observed from the results, shown in Table 3, that the adsorption capacity of carbon A towards PNP and MAP is higher in comparison with the uptake of the oxidized carbon B, which shows that the determining factors for their adsorption properties are the porous parameters and the chemical character surface area..

To counterbalance the effect of the different porous features of these carbons, the isotherms were normalized vs the surface area (see Figure 5). The differences in the uptake of PNP or MAP become less remarkable for both carbons, although still larger for PNP and carbon A, confirming that the adsorptive performance do not exclusively depend on the porosity, but it also depends on the chemical nature of the activated carbons, outlining the importance of the adsorbate's affinity. Indeed, the value bQo is larger in the hydrophobic carbon A, regardless the nature of the pollutant; for a specific carbon it is higher for MAP than PNP, despite the adsorption capacity of the latter is higher than the former for both studied carbons. The slightly higher uptake of PNP on both carbons is somewhat expected, implying that this compound displays a lower water solubility (1.7 vs $2.6 \mathrm{~g} / 100 \mathrm{~g}$ water for PNP and MAP, respectively). On the other hand, the trend observed for the affinity parameter seem to be related to the electron-withdrawing or electron-donating properties of the moieties present in the phenolic compound (i.e., nitro and amino groups). The $\mathrm{NH}_{2}$ - moiety is a powerful 
electron-donating group, that increases the $\pi$ electron density of the aromatic ring of the phenolic compound; in contrast, the $\mathrm{NO}_{2}$-moiety is a strong deactivator of the benzene ring. On the other hand, it is well known that oxygen functionalities withdraw the $\pi$-electron density of the basal planes of the carbons; thus, based on the literature, it seems reasonable to expect that the affinity of both aromatics would be higher in carbons with a rich $\pi$-electron density, and stronger for MAP which aromatic ring is not deactivated by the amino group [28, 29]. Our results indicate that both compounds have stronger affinity towards carbon A, despite this material has a relatively large amount of oxygen functionalities (Table 2), which are often considered as withdrawing groups (as above mentioned). Thus, it appears that despite the high oxygen content, deactivation of the aromatic ring is not very effective for carbon A (compared to its oxidized counterpart). This has to be attributed to the nature basic groups (i.e., ether and quinone-like structures) according to Boehm tritation.

Whereas previous studies reported in the literature $[28,29]$ deal exclusively with hydrophobic carbon adsorbents, disregarding the complexity of the adsorption process when hydrophilic materials are used (and thus water competition), our studies reveal the paramount importance of the carbon surface chemistry. In this regard, carbon A exhibits a strong interaction with PNP and MAP, despite its relatively large oxygen content (ca. 7.1 wt.\%) which is often associated to a large deactiviation effect of the $\pi$-electron density of the carbons graphene layers . Our results suggest that the content should not be considered as the key factor in the removal of aromatics; rather the basic/acidic nature of the surface functionalities should be taken into account.

\subsection{Effect of solution $\mathrm{pH}$}

Solution $\mathrm{pH}$ is one of the key factors that control the adsorption process, since it influences the electrostatic interactions between the adsorbent and the adsorbate. The adsorption of MAP and PNP as a function of $\mathrm{pH}$ was studied on both activated carbons over a $\mathrm{pH}$ range 2-12 (Figure 5). The desired $\mathrm{pH}$ was obtained by adding adequate amounts of $\mathrm{NaOH}$ or $\mathrm{HCl}$ diluted solutions to the initial non-buffered solution containing the phenolic compound. The uptake of both compounds was found to be maximum between 6-7 $\mathrm{pH}$ units, close to their respective $\mathrm{pK}_{\mathrm{a}}$ (i.e., 8.2 and 7.1 for MAP and PNP, respectively) for carbon $\mathrm{A}$ of a hydrophobic nature. For the oxidized carbon B, a different trend was obtained. The uptake is maximal at acidic $\mathrm{pH}$, and it gradually goes down as the solution becomes more basic. As expected, these results confirm that both aromatic compounds are preferentially adsorbed 
from their neutral form and that the effect of solution $\mathrm{pH}$ seems to depend to a large extent on the nature of the adsorbent [28].

In the case of PNP, the amount adsorbed appeared not to be too much altered at acidic $\mathrm{pH}$ (below 6), whereas the gradual shift at basic media resulted in a sharp fall of the uptake. As the $\mathrm{pH}$ increases, the surface of the activated carbons is being negatively charged until solution $\mathrm{pH}>\mathrm{pH}_{\mathrm{PZC}}$, where the amount of negative charges becomes predominant in the carbon surface. At this point, the fall in the uptake is due to the repulsive interactions, that appear between the anionic form of the adsorbates and the charges on the carbon surfaces. For the hydrophobic carbon $\mathrm{A}$, the occurrence of negative electrostatic interactions starts at $\mathrm{pH}$ beyond 8.2 units. On the other hand, negative charges are present on carbon $\mathrm{B}$ in a larger $\mathrm{pH}$ range (from $\mathrm{pH}$ close to 4 units). Hence, the uptake in the hydrophilic carbon is more sensitive to $\mathrm{pH}$, and adsorption become unfavored due to repulsive interaction when the ionization of the adsorbates becomes important (see speciation diagram in Figure 5). These results also explain that the affinity of both PNP and MAP (coefficient $b_{0}$ in Table 3) was larger for carbon A.

\section{Conclusions}

The results reported in this work show that mixtures of coal by-product (coal tar pitch) and furfural (material contained in biomass wastes) is appropriate raw materials for the synthesis of nanoporous carbons with negligible ash content and a well developed porosity. The synthetic activated carbon showed high adsorption activity towards phenolic derivates (MAP and PNP), comparable to those of other materials reported in the literature.

The adsorption capacities of the activated carbon and its oxidized modification towards phenolic derivatives (MAP and PNP) depended on the porous parameters and surface chemistry, the latter governing the retention mechanism. Non specific interactions are dominant for carbon $\mathrm{A}$, whereas for the oxidized adsorbent electrostatic interactions (repulsive and/or attractive) may appear depending on the solution $\mathrm{pH}$.

Our results outlined the paramount importance of the carbon surface chemistry on the removal of aromatics. Large oxygen contents are often associated to a large withdrawal effect of the pi-electron density of the graphene layers of carbon materials. However, our results suggest that the rather than the oxygen content, the basic/acidic nature of the surface functionalities should be taken into account. 


\section{Acknowledgement}

The authors thank to Ministry of Education, Youth and Science for financial support (grant number DO 02-222/17.12.2008). COA thanks the Spanish ministry for financial support (project CTM2008/01956 TECNO).

\section{References}

[1] V.Uberoi, S.K.Bhattacharya, Toxicity and degradability of nitrophenols in anaerobic systems. Water Environ Res. 69(1997) 146-156.

[2] K.Karim, S.K.Gupta, S.K., Biotransformation of nitrophenols in upflow anaerobic sludge blanket reactors. Bioresour Technol 8(2001) 179-186.

[3] R.M.Melgoza, G.Buitron, Degradation of p-nitrophenol in a batch biofilter under sequential anaerobic/aerobic environments. Water Sci Technol 44 (2001) 151-157.

[4.] EPA. Environmental Protection Agency. Ambient water quality for nitrophenols EPA. 1980;.440:580-063.

[5] Directive 2000/60/EC, EU Water Framework Directive. Ogg. J.L 327, 22 December 2000

[6] Directive 2008/105/EC Environmental quality standards for priority substances and certain other pollutants, 16 December 2008.

[7] H.Marsh, F. Rodriguez-Reinoso, In Activated Carbon, Elsevier Science 2006, Oxford, UK.

[8] N. Petrov, T. Budinova, Oxidized carbon from polyolefine wax. Chemie Ingenieur Technic 64 (1992) 64- 66.

[9] N. Petrov, T. Budinova, M. Razvigorova,, E. Ekinci, F. Yardim, V. Minkova, Preparation and characterization of carbon adsorbents from furfural, Carbon 38 (2000), 2069-2075.

[10] D. Savova, E. Apak, E. Ekinci, F. Yardim, N. Petrov, T. Budinova, M. Razvigorova, V. Minkova, Biomass conversion to carbon adsorbents and gas, Biomass and Bioenergy 21 (2001)133-142.

[11] B.Petrova, B.Tsyntsarski, T.Budinova, N.Petrov, CO Ania, J.B.Parra, M.Mladenov, P.Tzvetkov, 2010.Synthesis of nanoporous carbons from mixtures of coal tar pitch and furfural and their application as electrode materials, Fuel Processing Technology 91(2010), 1710-1716.

[12] F. Rouquerol, J. Rouquerol, K. Sing, In Adsorption by powders and porous solids. Principles, methodology and applications, Academic Press, London, 1999.

[13] H.P.Boehm, Chemical Identification of Surface Groups. In: Eley,D.D., Pines, H., Weisz, P.B.(eds.), Advances in catalysis and Related Subjects, vol.16. Academic Press, New York, (1966) pp.179-274.

[14] E.Papier, S.Li, J.B.Donnet, Contribution to the study of basic surface gropus on carbons, Carbon, 25 (1987) 243.

[15] P.J.M.Carrott, M.M.L.Ribeiro-Carrott, R.P.Lima, Preparation of activated carbon 'membranes' by physical and chemical activation of cork. Carbon 37(1999), 515-517.

[16] A.S.Mestre, J.Pires, J.M.F.Nogueira, J.B. Parra, A.P.Carvalho, CO Ania, Waste-derived activated carbons for removal of ibuprofen from solution: Role of surface chemistry and pore structure, Bioresources Technology 100(2009) 1720-1726.

[17] B.Cabal, T.Budinova, CO.Ania, B.Tsyntsarski, J.B.Parra, B.Petrova, Adsorption of naphthalene from aqueous solution on activated carbons obtained from bean pods, Journal of Hazardous materials 161(2009) 1150-1156.

[18] Y.S.Ho, G.McKay, 1988.Sorption of dye from aqueous solution by peat. Chem. 
Eng. J. $70,115-124$.

[19] B. Cabal., C.O. Ania, J.B. Parra, J.J. Pis, Kinetics of naphthalene adsorption on an activated carbon: comparison between aqueous and organic media. Chemosphere 76(2009), 433-438,

[20] I.A.W. Tan, A.L. Ahmad, B.H. Hameed, Adsorption isotherms, kinetics, thermodynamics and desorption studies of 2,4,6-trichlorophenol on oil palm empty fruit bunch-based activated carbon. Journal of Hazardous Materials, 164(2009), 473-482.

[21] C.H. Giles, T.H. MacEwan, S.N. Nakhwa, D. Smith, , "Studies in adsorption. Part XI. A system of classification of solutions adsorption isotherms, and its use in diagnosis of adsorption mechanisms and in measurement of specific surface areas of solids". J. Chem. Soc. (1960) 3973-3993.

[22] C. Troca-Torrado, M. Alexandre-Franco, C. Fernández-González, M. AlfaroDomínguez, V. Gómez-Serrano, Development of adsorbents from used tire rubber. Their use in the adsorption of organic and inorganic solutes in aqueous solution, Fuel Processing Technol, 92(2)(2011), 206-212.

[23] C. Moreno-Castilla, J. Rivera-Utrilla, M.V. Lopez-Ramon, F. Carrasco-Marín, Adsorption of some substituted phenols on activated carbons from a bituminous coal, Carbon 33(1995), 845-851.

[24] I. Langmuir. The adsorption of gases on plane surface of glass, mica and platinum, J. Am. Soc. 409(1916) 161-168.

[25] A. Kumar, S. Kumar, D.V. Gupta, Adsorption of phenol and 4-nitrophenol on granular activated carbon in basal salt medium: Equilibrium and kinetics, Journal of Hazardous Materials, 147(2007) 155-166.

[26] M. Franz, H.A. Arafat, N.G. Pinto, Effect of chemical heterogeneity on the adsorption mechanism of dissolved aromatics The determination of p-nitrophenol adsorption isotherms on activated carbon. Carbon 38(2000), 1807-1819.

[27] S. Haydar, M.A. Ferro-Garcia, J. Rivera-Utrilla, J.P. Joly, Adsorption of p-nitrophenol on an activated carbon with different oxidations, Carbon 41(2003), 387-395.

[28] C.Moreno-Castilla, J. Rivera-Utrilla, M.V.Lopez-Ramon, F.Carrasco-Marín,1995, Adsorption of some substituted phenols on activated carbons from a bituminous coal, Carbon 33(1995), 845-851.

[29] F.Villacanas, M.F.R.Pereira, J.M.F.Orfao, J.L. Figueiredo, Adsorption of simple aromatic compounds on activated carbons, J. Colloid Interf. Sci., 293(2006), 128-136. 


\section{FIGURE CAPTIONS}

Figure 1. X-ray diffraction of the synthetic activated carbon (carbon A).

Figure 2. SEM photograph of synthetic activated carbon (carbon A).

Figure 3. $\mathrm{N}_{2}$ adsorption isotherms at $-196{ }^{\circ} \mathrm{C}$ of the studied carbons, and main textural parameters (inset).

Figure 4. Effect of contact time on adsorption of aminophenol: carbon A (a) and carbon B (b).

Figure 5. Equilibrium adsorption isotherms of PNP and MAP on the studied activated carbons, expressed in terms of the amount adsorbed per gram of adsorbent $[\mathrm{mg} / \mathrm{g}]$ and per surface area of the adsorbent $\left[\mathrm{microg} / \mathrm{m}^{2}\right]$, after normalization vs the BET surface area.

Figure 6. Effect of $\mathrm{pH}$ on the retention of PNP and MAP on carbon A and B. For clarity, the pH-specification diagrams of MAP and PNP have been included, with indication of the $\mathrm{pH}$ values of carbon A (dashed vertical line) and carbon $\mathrm{B}$ (dotted vertical line). 


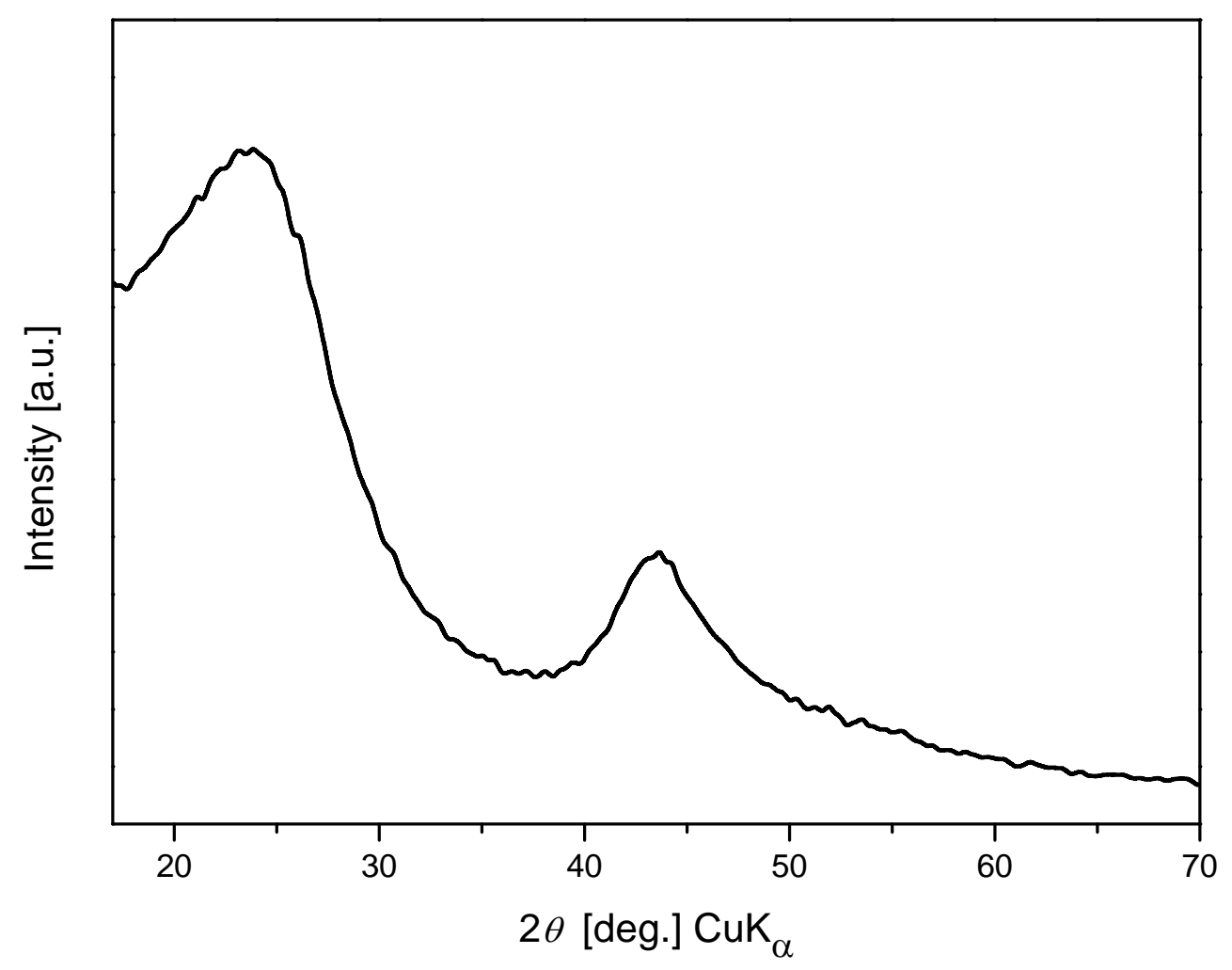

Fig. 1 


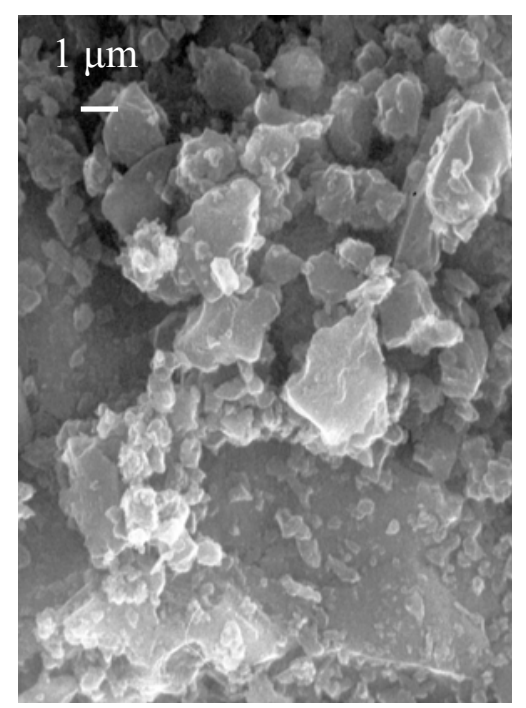

Fig. 2 


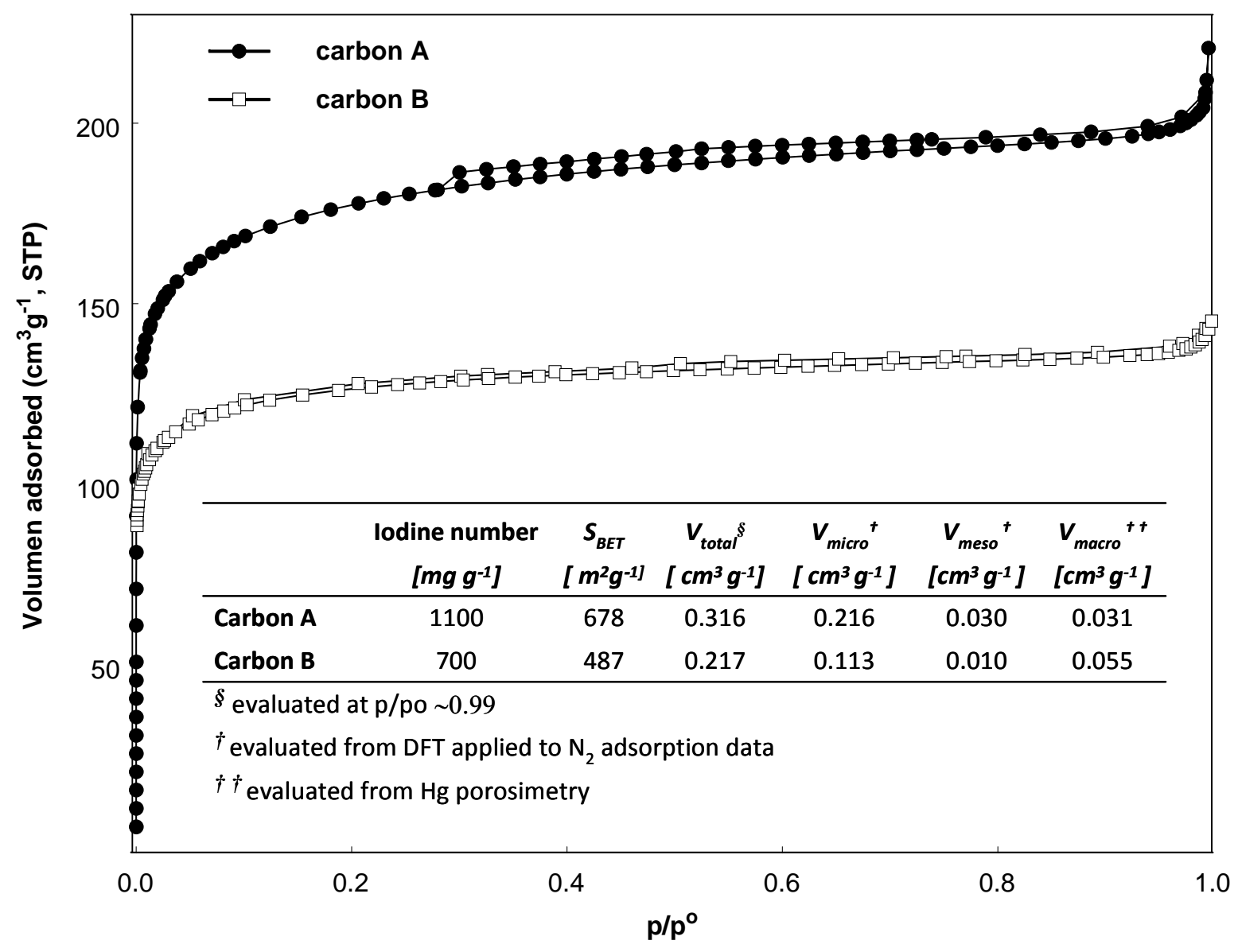

Fig. 3 

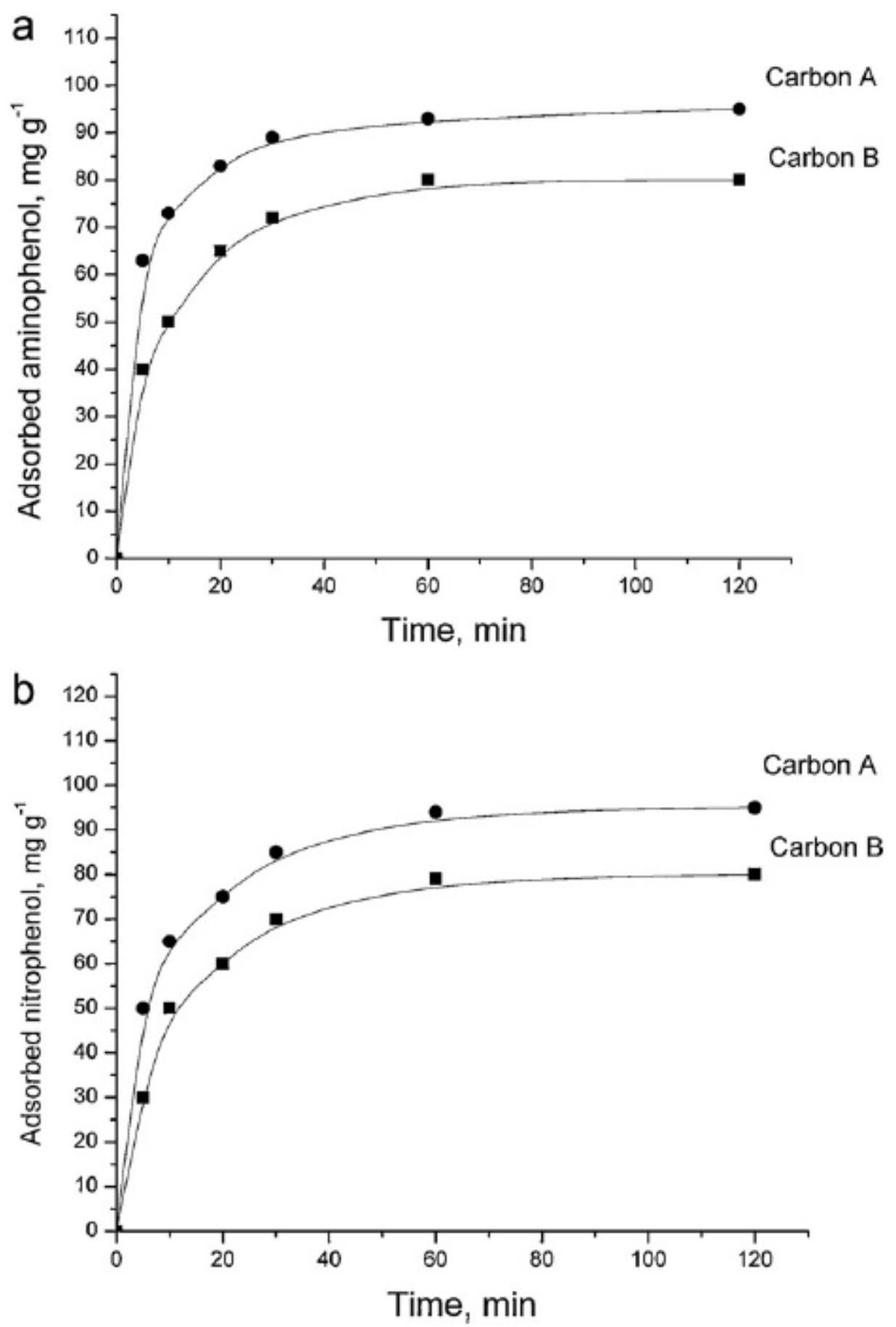

Fig. 4 

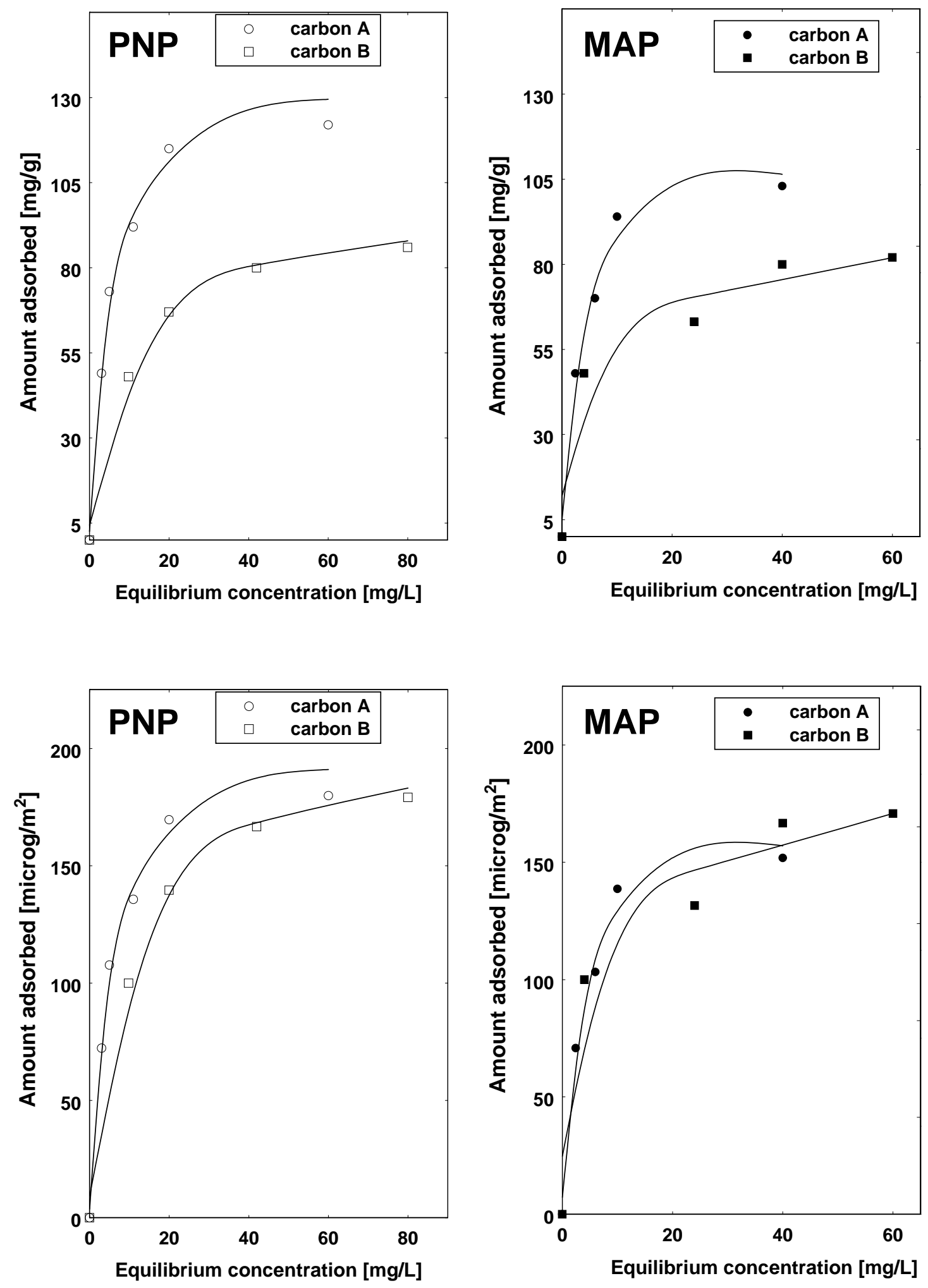

Fig. 5 


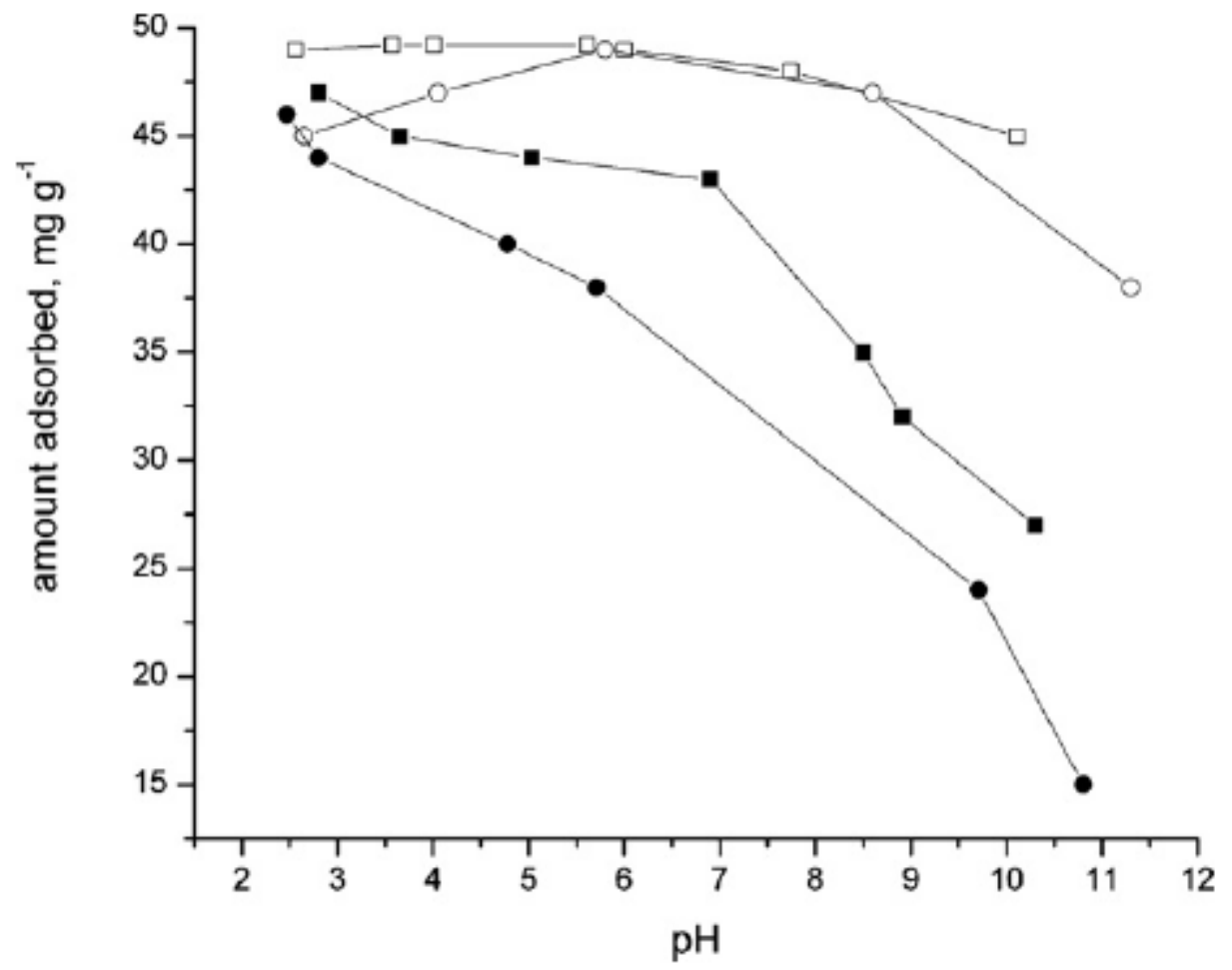

Fig. 6

Table 1. Chemical composition and $\mathrm{pH}$ value of the coal tar pitch and the synthesized activated carbons (wt.\%)

\begin{tabular}{llllllll}
\hline Sample & Ash & Volatiles (daf) & C & H & N & S & O \\
\hline Coal tar pitch & - & - & 90.90 & 4.95 & 0.90 & 0.50 & 2.75 \\
\hline Carbon A & 0.8 & 1.9 & 90.81 & 0.82 & 0.75 & 0.51 & 7.11 \\
\hline Carbon B & 1.5 & 7.7 & 86.77 & 1.02 & 1.30 & 0.56 & 10.35 \\
\hline
\end{tabular}

Table 2. Data corresponding to Boehm's titration for the quantification and identification of the oxygen surface groups on the activated carbons [meq $\left.\mathrm{g}^{-1}\right]$.

\begin{tabular}{lllllll}
\cline { 2 - 6 } Sample & Carboxylic & Lactonic & Phenolic & Carbonyl & Basic groups & pH \\
\hline Carbon A & BDL* & BDL & 0.210 & 1.356 & 0.778 & 8.1 \\
\hline Carbon B & 0.109 & 0.239 & 0.456 & 1.767 & 0.330 & 3.7 \\
\hline
\end{tabular}

*BDL - below detection limit 
Table 3. Results obtained from Langmuir equation applied to the adsorption isotherms of phenolic compounds on the studied activated carbons.

\begin{tabular}{|c|c|c|c|c|c|}
\hline Sample & & $\begin{array}{l}Q_{0} \\
{\left[\mathrm{mg} \mathrm{g}^{-1}\right]}\end{array}$ & $\begin{array}{l}\text { b } \\
{\left[1 \mathrm{mg}^{-1}\right]}\end{array}$ & $\begin{array}{l}\mathbf{b Q}_{0} \\
{\left[\mathbf{l} \mathbf{g}^{-1}\right]}\end{array}$ & $\mathbf{R}^{2}$ \\
\hline Carbon A & MAP & 110 & 0.53 & 58.7 & 0.994 \\
\hline Carbon A & PNP & 132 & 0.22 & 29.0 & 0.999 \\
\hline Carbon B & MAP & 85 & 0.48 & 42.7 & 0.969 \\
\hline Carbon B & PNP & 97 & 0.11 & 10.5 & 0.985 \\
\hline
\end{tabular}

